\title{
Safety and efficacy of the addition of simvastatin to cetuximab in previously treated $K R A S$ mutant metastatic colorectal cancer patients
}

\author{
J. M. Baas ${ }^{1}$ - L. L. Krens ${ }^{2}$ - A. J. ten Tije ${ }^{3}$ F. Erdkamp ${ }^{4}$ - T. van Wezel ${ }^{5}$ - H. Morreau ${ }^{5}$. \\ H. Gelderblom ${ }^{1}$ - H. J. Guchelaar ${ }^{2}$
}

Received: 4 May 2015 / Accepted: 27 August 2015 /Published online: 19 September 2015

(C) The Author(s) 2015. This article is published with open access at Springerlink.com

\begin{abstract}
Summary Introduction Cetuximab is registered for use in colorectal cancer (CRC) patients with $R A S$ wild-type tumours only. Simvastatin blocks the mevalonate pathway and thereby interferes with the post-translational modification (prenylation) of KRAS. We hypothesize that the activitated KRAS pathway in KRAS mutant tumors can be inhibited by simvastatin rendering these tumors sensitive to the EGFR inhibitor cetuximab. Methods A Simon two-stage, single-arm, phase II study was performed to test the efficacy and safety of the addition of simvastatin to cetuximab in patients with a $K R A S$ mutation in their CRC tumour who were previously treated with fluoropyrimidine, oxaliplatin and irinotecan based regimens. The primary endpoint was to test the percentage of patients alive and free from progression 12.5 weeks after the first administration of cetuximab. Our hypothesis was that at least $40 \%$ was free from progression, comparable to, though slightly lower than in KRAS wild-type patients. Results Four of 18 included patients $(22.2 \%)$ were free from progression at the primary endpoint time. The time to
\end{abstract}

J. M. Baas and L. L. Krens contributed equally to this work.

J. M. Baas

j.m.baas@lumc.nl

1 Department of Clinical Oncology, Leiden University Medical Center, Albinusdreef 2, P.O. Box 9600, 2300 RC Leiden, The Netherlands

2 Department of Clinical Pharmacy and Toxicology, Leiden University Medical Center, Albinusdreef 2, 2300 RC Leiden, The Netherlands

3 Department of Clinical Oncology, Amphia Hospital, Langendijk 75, 4819 EV Breda, The Netherlands

4 Department of Clinical Oncology, Orbis Medical Center, Dr. H. van der Hoffplein 1, 6162 BG Sittard-Geleen, The Netherlands

5 Department of Pathology, Leiden University Medical Center, Albinusdreef 2, 2300 RC Leiden, The Netherlands progression in these 4 patients ranged from 20.3 to 47 weeks. Conclusion Based on the current study we conclude that the theoretical concept of KRAS modulation with simvastatin was not applicable in the clinic, as we were not able to restore sensitivity to cetuximab in CRC patients harbouring a somatic KRAS mutation.

Keywords $K R A S \cdot$ Colorectal cancer $\cdot$ Cetuximab $\cdot$ Statin

\section{Introduction}

Each year over 940.000 patients are diagnosed with colorectal cancer (CRC) world-wide and over 500.000 people die of this disease [1]. In patients with advanced or metastatic colorectal treatment with monoclonal antibodies directed against the epidermal growth factor receptor (EGFR), cetuximab and panitumumab are proven to be active after failing fluoropyrimidine, oxaliplatin and irinotecan based regimens, though only in patients with tumours without a mutation in the $K R A S[2,3]$ or more recently $R A S$ gene [4]. This led to the question whether increased activation of KRAS signaling by $K R A S$ mutations can be modulated, thereby making KRAS mutated tumours sensitive to EGFR inhibitor therapy. One possible target for modulation is the mevalonate pathway, as we have previously discussed [5].

The mevalonate pathway is a metabolic cascade with various end-products including cholesterol. Other end-products are farnesyl and geranylgeranyl moieties (C15 and C17), both essential for posttranslational prenylation of the RAS protein and its association with the cytoplasmic membrane, and thereby activation of the RAS protein. By using HMG-CoA reductase inhibitors not only the synthesis of cholesterol is inhibited, but also the formation of $\mathrm{C} 15$ and $\mathrm{C} 17$, thereby inhibiting posttranslational modification of RAS [5, 6]. By 
blocking the mevalonate pathway in CRC patients with $K R A S$ mutated tumours, the activated KRAS pathway might be inhibited. This would theoretically lead to increased sensitivity to cetuximab, potentially comparable to tumours with wild-type $K R A S$.

This single-arm, phase II study was designed to test the safety and efficacy of the addition of simvastatin to cetuximab in patients with a KRAS mutation in their tumour who were previously treated with fluoropyrimidine, oxaliplatin and irinotecan based regimens.

\section{Methods}

\section{Patients}

Eligible patients had advanced or metastatic colorectal cancer with a mutation in codon 12, 13 or 61 of the $K R A S$ gene (either on tissue of the primary tumour or of a metastasis), after failing fluoropyrimidine, oxaliplatin and irinotecan based regimens, or after failure of oxaliplatin based therapy in patients who cannot be treated with irinotecan.

Other eligibility criteria included age 18 years or older, written informed consent, World Health Organisation (WHO) performance score of 0 to 2 and progression of disease in the past 3 months prior to inclusion. Exclusion criteria included symptomatic brain metastases, previous treatment with EGFR inhibitors and history of toxicity during statin.

The study protocol was approved by the Ethics Committees of all participating hospitals.

\section{Study design}

This phase II, single-arm, multi-center study was performed using a Simon two-stage design [7]. In the first stage, 15 patients were included, followed by an interim analysis. Results of this analysis would determine whether the combination of simvastatin and cetuximab may have clinical benefit in this group of CRC patients, thus justifying the second stage and including up to 41 patients.

\section{Treatment schedule}

Cetuximab was first administered at least one week after start of simvastatin therapy. The initial cetuximab dose was $400 \mathrm{mg} / \mathrm{m}^{2}$ with subsequent weekly infusions of $250 \mathrm{mg} / \mathrm{m}^{2}$. Pretreatment with an antihistamine and a corticosteroid was mandatory before the first infusion of cetuximab and recommended for all subsequent infusions.

Simvastatin $80 \mathrm{mg}$ orally once daily was started at start of study participation. This dose was chosen taken into consideration the need for continuous administration of the statin during the entire study, inhibitory effect on the mevalonate pathway and tolerability. Statins in cancer therapy have been studied in clinical trials in solid [8-18] and haematologic [19-21] malignancies, both as monotherapy as well as additional to chemotherapy. Statin doses from $20 \mathrm{mg}$ /day up to $35 \mathrm{mg} / \mathrm{kg} /$ day were used, with only continuous use of statins when dosed at a maximum of $80 \mathrm{mg} /$ day. Since the aim of this study is to modulate KRAS during the entire treatment with cetuximab and therefore a continuous exposure to simvastatin is needed, a dose of $80 \mathrm{mg} /$ day was selected in order to obtain maximum effect while minimizing the risk of toxicity.

Treatment was continued until progression of disease, clinical signs of progression, unacceptable toxicity or cetuximab toxicity requiring withholding of more than 2 subsequent infusions.

Tumour response was every 6 weeks using CT-scans and according to Response Evaluation Criteria In Solid Tumors (RECIST) version 1.1. Scans of patients free from progression at time of primary endpoint were centrally reviewed.

\section{Endpoints}

Primary objective was to test the percentage of patients alive and free from progression and alive at 12.5 weeks after the first administration of cetuximab. Our hypothesis was that at least $40 \%$ of patients was free from progression, comparable to though slightly lower than in $K R A S$ wild-type patients [2].

Secondary objectives were to investigate overall survival (OS), objective response rate (ORR), progression free survival (PFS), and safety of simvastatin combined with cetuximab in this population and to evaluate the correlation between skin toxicity and response to treatment. Exploratory endpoints were to investigate the role of cholesterol as a biomarker during this treatment and whether PIK3CA status correlates with response to cetuximab in this population.

\section{Mutational analysis}

$K R A S$ mutational status was reconfirmed centrally, testing for the 7 most frequent mutations in codon 12 and 13 as described in detail elsewhere [22]. In addition, we tested for the 3 most common mutation in the PIK3CA gene; in exon 9 (c.1624G $>$ A (p.E542K) and c.1633G $>$ A (pE545K)) and exon 20 (c.3140A $>$ G (p.H1047R)). Though KRAS and BRAF mutations are known to be mutually exclusive [23], we did test for the activating hotspot mutation p.V600E.

\section{Statistics}

Sample size was chosen based on previous published data of CRC patients with KRAS wild-type tumours treated with cetuximab [2], aiming for a at least $40 \%$ of patients free from progression at 12.5 weeks after start of cetuximab treatment in patients with $K R A S$ mutant type tumours (i.e., slightly lower 
than the effect in $K R A S$ wild-type patients). Combined with an alpha of 0.05 and a power of 0.80 , an interim size of 15 and a total sample size of 46 patients were required. An interim analysis was to be performed after the inclusion of 15 evaluable patients. Only when at least $40 \%$ (i.e., 6 patients) were free from progression at the 12.5 weeks, another 31 patients would be enrolled during the second stage of the study.

\section{Results}

\section{Patients}

During the first stage of the study 18 instead of 15 patients were enrolled to account for patients that were thought to unevaluable for the primary endpoint. Baseline characteristics are listed in Table 1. None of the patients were using statins prior to inclusion.

\section{Efficacy}

Four of 18 patients were free from progression at the primary endpoint time, therefore the percentage of patients alive and free from progression 12.5 weeks after the first administration of cetuximab was $22 \%$. The time to progression in these 4 patients ranged from 20.3 to 47 weeks. Drug exposure to simvastatin and cetuximab was equal for all patients.

Figure 1 shows progression free (panel A) and overall survival (panel B). Median PFS was 9 weeks (mean 12.9 weeks, range 3.9-47 weeks). Median OS was 31.5 weeks (mean 36.3,

Table 1 Baseline characteristics range $8-138.1$ ). The ORR was $6 \%$ (partial remission in 1 patient). A true relation between skin toxicity and efficacy of treatment was not observed in this study though this may (partly) be due to the low number of patients and due to the improved knowledge of the efficacy of pre-emptive skin toxicity management.

\section{Safety}

Main symptoms and adverse events reported on study reported were fatigue $(n=11)$, acne $(n=10)$ and rash $(n=6)$. Myopathy was not reported. Three patients had elevation of creatine kinase (CK) levels on study (grade 4 in one patient). Skin toxicity occurred in 10 patients; the worst grade of acneiform rash was grade 3 in one patient, grade 2 in 4 patients and grade 1 in the remaining 5 patients. One patient experienced a severe (i.e., grade 3) allergic reaction during the first infusion of cetuximab. One of the serious adverse events did precede the death of a participant. Upon the scheduled laboratory examination severe elevation of liver enzymes were observed. Rhabdomyolysis was considered, (though on study CK levels were below $3.000 \mathrm{U} / \mathrm{l}$ ) and so was progression of liver metastases. Study medication was interrupted immediately, however the patient's situation did not improve and it was decided to terminate study participation permanently. Specific SNPs associated with increased risk of statininduced myopathy (i.e., SLCO1B1 variants [24]) were considered though none were identified in this patient. The patient deceased few weeks later. Post-mortem examination did not occur.

\section{Exploratory endpoints}

All patients showed cholesterol reduction, ranging from a maximum reduction of 0.8 to $64.4 \%$. The percentage of cholesterol reduction did not correlate with progression free survival.

Tumour tissue of 15 patients was available for central review. Table 2 shows mutational status of $K R A S$ and PIK3CA per patient. Of the 4 patients responding to treatment, 3 had a KRAS mutation in codon 12 and 1 had a PIK3CA mutation. As expected all patients were $B R A F$ wild-type.

\section{Discussion}

To our knowledge, this is the first clinical trial testing the addition of simvastatin to cetuximab monotherapy in CRC patients harbouring a KRAS mutation in tumour tissue as an attempt to restore cetuximab sensitivity. While it was remarkable to notice a durable progression free survival in 4 patients, the interim analysis showed that the predefined criteria to proceed to the second stage of this study were not reached. 
Fig. 1 a Progression free survival in weeks for the addition of simvastatin to cetuximab in CRC patients failing standard therapy. b Overall survival in weeks for the addition of simvastatin to cetuximab in CRC patients failing standard therapy
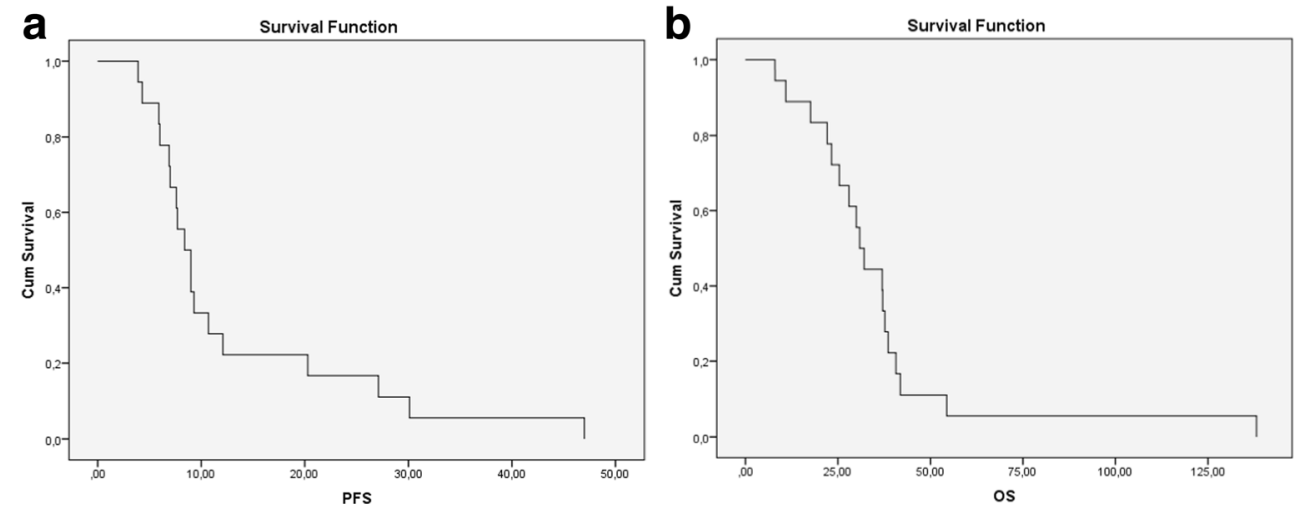

Therefore, the current study suggests that high dose simvastatin does not render cetuximab sensitivity in KRAS mutant CRC.

Statines are one of several potential agents to modulate KRAS signaling, as we have previously reviewed [5]. The current study is not the first to hypothesize on statins and their inhibitory effect on the activity of RAS and its downstream pathway. However, all but one previous reports include only preclinical data. For example, lovastatin and simvastatin inhibit downstream activity in breast cells with mutated $H R A S$, possibly by inhibiting membrane localization of HRAS. The effect was reversed when adding farnesyl pyrophosphate, indicating the effect was related to prenylation of RAS [25]. More recently, simvastatin was shown to restore cetuximab resistance in vitro and in vivo [26]. Based on these results, one might wonder whether the negative outcome of the current study would have been different if using higher doses of

Table $2 K R A S$ and $P I K 3 C A$ mutational status per patient

\begin{tabular}{lll}
\hline Study number & KRAS mutation & PIK3CA mutational status \\
\hline 1 & G12D & Wild-type \\
2 & G12V & Wilde-type \\
3 & G12V & Wild-type \\
4 & G12C & Wild-type \\
5 & G12V & Wild-type \\
6 & G12S & Wild-type \\
7 & missing & Missing \\
8 & G12V & Wild-type \\
9 & G13D & Wild-type \\
10 & G13D & Wild-type \\
11 & G12D & Wild-type \\
12 & G12D & Wild-type \\
13 & missing & Missing \\
14 & G12V & Wild-type \\
15 & G12A & Wild-type \\
16 & G12A & Wild-type \\
17 & G13D & Mutation in exon 9 \\
18 & G12D & Mutation in exon 9 \\
\hline
\end{tabular}

simvastatin. However, preclinical data showed a significant reduction in cell growth of $K R A S$ mutant CRC cell lines using $0.2 \mu \mathrm{M}$ simvastatin, the equivalent of $2 \mathrm{mg} / \mathrm{kg} /$ day in humans [26]. Moreover, in cardiovascular disease the registered dose of $80 \mathrm{mg}$ of simvastatin is significantly lowers cholesterol serum levels. It is reasonable that this dose will also affect the formation of the $\mathrm{C} 15$ and $\mathrm{C} 17$ groups and subsequently the prenylation of the KRAS protein. Furthermore, we question whether higher doses will be feasible in terms of safety.

A recent study of Lee et al. tested the efficacy of the addition of the same dose of simvastatin (i.e., $80 \mathrm{mg}$ once daily) to cetuximab and irinotecan in KRAS mutant CRC patients failing prior oxaliplatin, fluoropyrimidine and irinotecan based therapy [27]. The initially reported PFS and OS (median 7.6 months and 12.8 months respectively) were considerably higher than historical results in chemotherapy refractory CRC patients with KRAS mutated tumours [28] and chemotherapy refractory CRC patients in general [29-31]. Moreover, these results were in contrast with our findings. However, a recent erratum published by this group showed that initial survival data were incorrect [32]. The corrected PFS and OS are in line with our results, providing no evidence for a modulating effect of simvastatin on the KRAS mutant phenotype.

The majority of patients had a KRAS mutation in codon 12 and only 3 in codon 13 . It has been reported that tumours harbouring a G13D mutation in the KRAS gene might be sensitive to EGFR-inhibitors [33]. Moreover, none of our patients had a PIK3CA mutation in exon 20, while these might also be more likely to be sensitive to EGFR-inhibitors, contrary to mutations in exon 9 [34]. However, of the 4 patients who were free from progression at time of the primary endpoint only one patients had a G13D mutation in the KRAS gene and none had a $P I K 3 C A$ mutation in exon 20 .

\section{Conclusion}

Based on the current study we conclude that the concept of $K R A S$ modulation with simvastatin was not applicable in the clinic. Similar results were recently demonstrated in this 
population treated with panitumumab and simvastatin [35]. Better treatment strategies are needed for this patient population.

\section{Compliance with ethical standards}

Conflict of interest Jara M, Baas: none; Lisanne L. Krens: none; A.J. ten Tije: none; F. Erdkamp: none; Tom van Wezel: none; Hans Morreau: none; Henk-Jan Guchelaar: research funding by Amgen Inc and Merck BV; Hans Gelderblom: research funding by Amgen Inc en Merck BV.

Informed consent The informed consent form was signed by the patient (and physician) prior to inclusion and according to the ICH guidelines on Good Clinical Practice.

Funding This was an Investigator Initiated Study, made possible by a research grant offered by Merck Serono.

Open Access This article is distributed under the terms of the Creative Commons Attribution 4.0 International License (http:// creativecommons.org/licenses/by/4.0/), which permits unrestricted use, distribution, and reproduction in any medium, provided you give appropriate credit to the original author(s) and the source, provide a link to the Creative Commons license, and indicate if changes were made.

\section{References}

1. Denters MJ, Deutekom M, Fockens P, Bossuyt PM, Dekker E (2009) Implementation of population screening for colorectal cancer by repeated fecal occult blood test in the Netherlands. BMC Gastroenterol 9:28

2. Karapetis CS, Khambata-Ford S, Jonker DJ et al (2008) K-ras mutations and benefit from cetuximab in advanced colorectal cancer. $\mathrm{N}$ Engl J Med 359:1757-1765

3. Amado RG, Wolf M, Peeters M et al (2008) Wild-type KRAS is required for panitumumab efficacy in patients with metastatic colorectal cancer. J Clin Oncol 26:1626-1634

4. Sorich MJ, Wiese MD, Rowland A, Kichenadasse G, McKinnon RA, Karapetis CS (2014) Extended RAS mutations and anti-EGFR monoclonal antibody survival benefit in metastatic colorectal cancer: a meta-analysis of randomized, controlled trials. Ann Oncol 26:13-21

5. Krens LL, Baas JM, Gelderblom H, Guchelaar HJ (2010) Therapeutic modulation of k-ras signaling in colorectal cancer. Drug Discov Today 15:502-516

6. Swanson KM, Hohl RJ (2006) Anti-cancer therapy: targeting the mevalonate pathway. Curr Cancer Drug Targets 6:15-37

7. Simon R (1989) Optimal two-stage designs for phase II clinical trials. Control Clin Trials 10:1-10

8. Hong JY, Nam EM, Lee J et al (2014) Randomized double-blinded, placebo-controlled phase II trial of simvastatin and gemcitabine in advanced pancreatic cancer patients. Cancer Chemother Pharmacol 73:125-130

9. Manoukian GE, Tannir NM, Jonasch E, Qiao W, Haygood TM, Tu SM (2011) Pilot trial of bone-targeted therapy combining zoledronate with fluvastatin or atorvastatin for patients with metastatic renal cell carcinoma. Clin Genitourin Cancer 9:81-88

10. Han JY, Lim KY, Yu SY, Yun T, Kim HT, Lee JS (2011) A phase 2 study of irinotecan, cisplatin, and simvastatin for untreated extensive-disease small cell lung cancer. Cancer 117:2178-2185

11. Han JY, Lee SH, Yoo NJ et al (2011) A randomized phase II study of gefitinib plus simvastatin versus gefitinib alone in previously treated patients with advanced non-small cell lung cancer. Clin Cancer Res 17:1553-1560

12. Konings IR, van der Gaast A, van der Wijk LJ, de Jongh FE, Eskens FA, Sleijfer S (2010) The addition of pravastatin to chemotherapy in advanced gastric carcinoma: a randomised phase II trial. Eur J Cancer 46:3200-3204

13. Lee J, Jung KH, Park YS et al (2009) Simvastatin plus irinotecan, 5fluorouracil, and leucovorin (FOLFIRI) as first-line chemotherapy in metastatic colorectal patients: a multicenter phase II study. Cancer Chemother Pharmacol 64:657-663

14. Graf H, Jungst C, Straub G et al (2008) Chemoembolization combined with pravastatin improves survival in patients with hepatocellular carcinoma. Digestion 78:34-38

15. Knox JJ, Siu LL, Chen E et al (2005) A Phase I trial of prolonged administration of lovastatin in patients with recurrent or metastatic squamous cell carcinoma of the head and neck or of the cervix. Eur J Cancer 41:523-530

16. Lersch C, Schmelz R, Erdmann J et al (2004) Treatment of HCC with pravastatin, octreotide, or gemcitabine - a critical evaluation. Hepatogastroenterology 51:1099-1103

17. Kim WS, Kim MM, Choi HJ et al (2001) Phase II study of highdose lovastatin in patients with advanced gastric adenocarcinoma. Invest New Drugs 19:81-83

18. Kawata S, Yamasaki E, Nagase T et al (2001) Effect of pravastatin on survival in patients with advanced hepatocellular carcinoma. A randomized controlled trial. Br J Cancer 84:886-891

19. Ahmed TA, Hayslip J, Leggas M (2013) Pharmacokinetics of highdose simvastatin in refractory and relapsed chronic lymphocytic leukemia patients. Cancer Chemother Pharmacol 72:1369-1374

20. Hus M, Grzasko N, Szostek M et al (2011) Thalidomide, dexamethasone and lovastatin with autologous stem cell transplantation as a salvage immunomodulatory therapy in patients with relapsed and refractory multiple myeloma. Ann Hematol 90:1161-1166

21. van der Spek E, Bloem AC, Sinnige HA, Lokhorst HM (2007) High dose simvastatin does not reverse resistance to vincristine, adriamycin, and dexamethasone (VAD) in myeloma. Haematologica 92:e130-e131

22. van Eijk R, Licht J, Schrumpf M et al (2011) Rapid KRAS, EGFR, BRAF and PIK3CA mutation analysis of fine needle aspirates from non-small-cell lung cancer using allele-specific qPCR. PLoS One 6: e17791

23. Tol J, Nagtegaal ID, Punt CJ (2009) BRAF mutation in metastatic colorectal cancer. N Engl J Med 361:98-99

24. Link E, Parish S, Armitage J et al (2008) SLCO1B1 variants and statininduced myopathy — a genomewide study. N Engl J Med 359:789-799

25. Kang S, Kim ES, Moon A (2009) Simvastatin and lovastatin inhibit breast cell invasion induced by H-Ras. Oncol Rep 21:1317-1322

26. Lee J, Lee I, Han B et al (2011) Effect of simvastatin on cetuximab resistance in human colorectal cancer with KRAS mutations. J Natl Cancer Inst 103:674-688

27. Lee J, Hong YS, Hong JY et al (2014) Effect of simvastatin plus cetuximab/irinotecan for KRAS mutant colorectal cancer and predictive value of the RAS signature for treatment response to cetuximab. Invest New Drugs 32:535-541

28. De Roock W, Claes B, Bernasconi D et al (2010) Effects of KRAS, BRAF, NRAS, and PIK3CA mutations on the efficacy of cetuximab plus chemotherapy in chemotherapy-refractory metastatic colorectal cancer: a retrospective consortium analysis. Lancet Oncol 11:753-762

29. Grothey A, van Cutsem E, Sobrero A et al (2013) Regorafenib monotherapy for previously treated metastatic colorectal cancer (CORRECT): an international, multicentre, randomised, placebocontrolled, phase 3 trial. Lancet 381:303-312

30. Jonker DJ, O'Callaghan CJ, Karapetis CS et al (2007) Cetuximab for the treatment of colorectal cancer. N Engl J Med 357:2040-2048 
31. van Cutsem E, Peeters M, Siena S et al (2007) Open-label phase III trial of panitumumab plus best supportive care compared with best supportive care alone in patients with chemotherapy-refractory metastatic colorectal cancer. J Clin Oncol 25:1658-1664

32. Lee J, Hong YS, Hong JY et al (2014) Erratum to: effect of simvastatin plus cetuximab/irinotecan for KRAS mutant colorectal cancer and predictive value of the RAS signature for treatment response to cetuximab. Invest New Drugs

33. Tejpar S, Celik I, Schlichting M, Sartorius U, Bokemeyer C, Van CE (2012) Association of KRAS G13D tumor mutations with outcome in patients with metastatic colorectal cancer treated with first-line chemotherapy with or without cetuximab. J Clin Oncol 30: 3570-3577

34. Karapetis CS, Jonker D, Daneshmand M et al (2014) PIK3CA, BRAF, and PTEN status and benefit from cetuximab in the treatment of advanced colorectal cancer-results from NCIC CTG/ AGITG CO.17. Clin Cancer Res 20:744-753

35. Baas JM, Krens LL, Bos MM et al (2015) Safety and efficacy of the addition of simvastatin to panitumumab in previously treated KRAS mutant metastatic colorectal cancer patients. Anticancer Drugs 26:872-877 